\title{
Four-Loop Gauge and Three-Loop Yukawa Beta Functions in a General Renormalizable Theory
}

\author{
Alexander Bednyakov $\circledast^{1,2, *}$ and Andrey Pikelner $\circledast^{1, \dagger}$ \\ ${ }^{1}$ Bogoliubov Laboratory of Theoretical Physics, Joint Institute for Nuclear Research, Dubna 141980, Russia \\ ${ }^{2}$ P. N. Lebedev Physical Institute of the Russian Academy of Sciences, Moscow 119991, Russia
}

(Received 25 May 2021; accepted 17 June 2021; published 20 July 2021)

\begin{abstract}
We present the beta functions of gauge and Yukawa couplings in general four-dimensional quantum field theory, at four and three loops, respectively. The essence of our approach is fixing unknown coefficients in the most general ansatz for beta functions by direct calculation in several simplified models. We apply our results to the standard model and its extension with an arbitrary number of Higgs doublets and provide expressions for all four-loop gauge couplings beta functions with matrix Yukawa interactions.
\end{abstract}

DOI: 10.1103/PhysRevLett.127.041801

Introduction.-Renormalization group equations (RGE) find their numerous applications in many physical problems formulated in the language of quantum field theory (QFT). Being a convenient tool to improve perturbation theory results, RGE allow one to obtain high-precision predictions for various quantities ranging from critical exponents in the theory of critical phenomena to observables in the standard model (SM) and its extensions.

In the early 1980s, Machacek and Vaughn [1-3] presented their two-loop RG functions in $\overline{\mathrm{MS}}$ scheme for all dimensionless couplings in general four-dimensional renormalizable QFT. Several misprints have been corrected during the subsequent 35 years, and the classical result has been extended to include RG functions for dimensionful parameters [4-7]. The two-loop general expressions became highly demanded in studies of the SM extensions. After the appearance of computer codes aimed to generate RGE for user-specified models, two-loop RG studies have become de facto standard in a new physics analysis.

After discovering the Higgs boson at the LHC $[8,9]$ and a burst of activity on the vacuum stability problem (see, e.g., [10]), it became clear that three-loop RG functions [11-16] can play an essential role in precision studies of the SM and its extensions. Partial four-loop results in the SM [17-19] and three-loop beta functions in the two-Higgs-doublet model (THDM) [20] became available during the past few years.

Recently, two major steps toward general high-order results have been made. First of all, RG functions in general theories were represented by a linear combination of

Published by the American Physical Society under the terms of the Creative Commons Attribution 4.0 International license. Further distribution of this work must maintain attribution to the author(s) and the published article's title, journal citation, and DOI. Funded by SCOAP. independent tensor structures (TS) corresponding to contractions of various indices. One can match these "template" expressions to known results in specific models and extract model- independent coefficients [21,22]. Second, new ideas based on the so-called Weyl consistency conditions (WCC) [23-25] allow one to find relations between known and unknown TS coefficients, thus, putting constraints on to-be-computed numbers. In particular, WCC relate gauge, Yukawa, and self-coupling beta functions computed at four, three, and two loops, respectively.

Let us also mention that WCC allows us to resolve [26] a well-known issue with $\gamma_{5}$ ambiguity (see, e.g., [27]) in $\overline{\mathrm{MS}}$ RG functions. Poole and Thomsen in Ref. [26] use WCC to relate the ambiguous four-loop terms in strong coupling beta function $[17,18]$ to unambiguous three-loop contributions to Yukawa beta functions [14,28] and confirm the prescription advocated in Ref. [17]. The relation holds for any four-loop gauge beta function in a general QFT, and it was immediately used for the gauge sector of the SM with diagonal Yukawa matrices [29].

There is also an (unphysical) ambiguity in RG functions due to possible unitary contributions $[20,30,31]$ to field renormalization in models with flavor symmetries. In what follows, we assume a natural choice of Hermitian anomalous dimension and refer to recent work [32] for more details.

In the current study, we present for the first time complete results for all TS coefficients in gauge and Yukawa beta functions at four and three loops, respectively. Partial results are already available in the literature $[21,22,25]$, and we want to emphasize the difference between our approach and methods used in previous studies. In the latter, given WCC, the unknown betafunction coefficients are fixed from known results for specific (usually physical) models. One of the most powerful constraints of this type is provided by the three-loop calculation in THDM [20]. 
On the contrary, we, for the first time, design simple toy models with specific gauge group structure, each giving constraints on different unknown coefficients. We use diagrams for TS provided in Supplemental Materials of Ref. [25] as a guide, but it is fair to say that we found the required models by trial and error. We use TSs implemented in the prominent code RGBeta [33] to speed up our investigation.

General four-dimensional QFT that covers most of possible phenomenological applications can be written in the form [25]

$$
\begin{aligned}
\mathcal{L}= & -\frac{1}{4} G_{A B}^{-2} F_{\mu \nu}^{A} F^{B \mu \nu}+\frac{1}{2}\left(D_{\mu} \phi\right)_{a}\left(D_{\mu} \phi\right)_{a}+\frac{i}{2} \Psi_{i}^{T} \gamma^{\mu}\left(D_{\mu} \Psi\right)_{i} \\
& -\frac{1}{2} \phi^{a} \Psi_{i}^{T} y_{a i j} \Psi_{j}-\frac{1}{24} \lambda_{a b c d} \phi_{a} \phi_{b} \phi_{c} \phi_{d}
\end{aligned}
$$

where $A, B$ runs through all factors of the gauge group $\mathcal{G}$. The coupling matrix $G_{A B}$ is symmetric and block diagonal with nondiagonal entries corresponding to kinetic mixing

$$
\beta_{A B}^{(4)}=\sum_{n=1}^{202}\left(\mathfrak{g}_{n}^{(4)} \cdot A n(n) n^{B}\right)
$$

with universal numerical coefficients $\mathfrak{g}_{n}^{(4)}$ and $\mathfrak{y}_{n}^{(3)}$. Our ultimate goal is to provide all these $202+308$ numbers explicitly.

Models.-We emphasize two different sources of constraints on beta-function coefficients. One type is coming from direct calculations and fixes independently $\mathfrak{g}^{(4)}$ and $\mathfrak{y}^{(3)}$. Another one is WCC providing relations between these coefficients. Most of the first type constraints, which one can obtain from SM and THDM, are already included in the analysis by Poole and Thomsen [25]. We extend these results by direct calculations in toy $\mathrm{SU}(n)$ gauge models described below. Calculation with arbitrary $n$ [34] gives an additional handle on unknown coefficients.

Because of the nature of constraints from WCC, it is natural to consider Gauge and Yukawa together. Since constraints from different sources are independent, the combined system becomes overdetermined, and we have a large number of equalities. The latter allows us not only to fix the TS coefficients but also to make extensive crosschecks of the RGBeta code and the validity of our results.

First of all, we consider an analog of scalar QCD with a gauge group $\mathcal{G}=\mathrm{SU}\left(n_{1}\right) \times \mathrm{SU}\left(n_{2}\right)$, in which a fundamental scalar $\phi$ is charged under both factors. The Lagrangian can be cast into the form between $\mathrm{U}(1)$ factors present in $\mathcal{G}$. The real scalars $\phi_{a}$ and Majorana fermions $\Psi_{i}$ belong to some representation of $\mathcal{G}$. The Yukawa couplings are denoted by $y_{a i j}$ and are symmetric in fermion indices $i j$. The self-coupling $\lambda_{a b c d}$ is symmetric in all four scalar indices.

In this Letter, we consider four-loop $\beta_{A B}^{(4)}$ and three-loop $\beta_{a i j}^{(3)}$ contributions to gauge and Yukawa beta functions, respectively. The latter are defined as

$$
\begin{aligned}
& \beta_{A B}=\frac{d G_{A B}^{2}}{d \ln \mu}=\frac{1}{2}\left[\sum_{l} G_{A C}^{2} \frac{\beta_{C D}^{(l)}}{(4 \pi)^{2 l}} G_{D B}^{2}+(A \leftrightarrow B)\right], \\
& \beta_{a i j}=\frac{d y_{a i j}}{d \ln \mu}=\frac{1}{2}\left[\sum_{l} \frac{\beta_{a i j}^{(l)}}{(4 \pi)^{2 l}}+(i \leftrightarrow j)\right],
\end{aligned}
$$

and can be represented in terms of 202 and 308 TSs [25], respectively,

$$
\beta_{a i j}^{(3)}=\sum_{n=1}^{308}(\mathfrak{y}_{n}^{(3)} \cdot i \underbrace{a}_{j})
$$

$$
\begin{aligned}
\mathcal{L}_{\mathbf{M} 1}= & -\frac{1}{2 g_{i}^{2}} \operatorname{Tr}\left(F_{\mu \nu}^{i} F_{\mu \nu}^{i}\right)+\left(D_{\mu} \phi\right)_{\alpha \rho}^{\dagger}\left(D_{\mu} \phi\right)_{\alpha \rho} \\
& -\frac{\lambda_{1}}{2}\left(\phi_{\alpha \rho}^{\dagger} \phi_{\alpha \rho}\right)\left(\phi_{\beta \sigma}^{\dagger} \phi_{\beta \sigma}\right)-\frac{\lambda_{2}}{2}\left(\phi_{\alpha \rho}^{\dagger} \phi_{\alpha \sigma}\right)\left(\phi_{\beta \sigma}^{\dagger} \phi_{\beta \rho}\right),
\end{aligned}
$$

where $g_{1}$ and $g_{2}$ are gauge couplings. To carry out renormalization, we need the self-interactions of scalars $\lambda_{1}, \lambda_{2}$ compatible with $\mathcal{G}$. In Eq. (4) we explicitly write group indices $\alpha, \beta$ and $\rho, \sigma$ corresponding to fundamental representations of $\mathrm{SU}\left(n_{1}\right)$ and $\mathrm{SU}\left(n_{2}\right)$, respectively.

The second model that we use is a gauge theory with single $\mathrm{SU}(n)$. The spectrum of the model consists of two fields in fundamental representation, a vectorlike Dirac fermion $Q$ and a scalar $h$, and two singlet fields, a Weyl spinor (in what follows, we use Dirac four-component spinors) $u_{R} \equiv P_{R} u$ and a scalar $s$ :

$$
\begin{aligned}
\mathcal{L}_{\mathbf{M} 2}= & -\frac{1}{4 g^{2}} F_{\mu \nu}^{a} F_{\mu \nu}^{a}+i \bar{Q} \gamma^{\mu} D_{\mu} Q+i \bar{u}_{R} \gamma^{\mu} \partial_{\mu} u_{R} \\
& +\frac{1}{2}\left(\partial_{\mu} s\right)^{2}+\left|D_{\mu} h\right|^{2}-y_{s} \bar{Q} Q s-y_{u}\left[(\bar{Q} h) u_{R}+\text { H.c. }\right] \\
& -\frac{\lambda_{s} s^{4}}{24}-\frac{\lambda_{s h}}{2} s^{2}\left(h^{\dagger} h\right)-\frac{\lambda_{h}}{2}\left(h^{\dagger} h\right)^{2} .
\end{aligned}
$$


Here $g, y_{s}$, and $y_{u}$ are gauge and Yukawa couplings of our interest, and $\lambda_{s}, \lambda_{s h}$, and $\lambda_{h}$ are the required scalar selfcouplings.

We also study a gauge theory with $\mathcal{G}=\mathrm{SU}\left(n_{1}\right) \times$ $\mathrm{SU}\left(n_{2}\right) \times \mathrm{SU}\left(n_{3}\right)$ describing interactions of a Dirac fermion $\Psi$ in fundamental representation of each factor in $\mathcal{G}$ and three adjoint scalars $\phi_{i}$, each charged only under one $\mathrm{SU}\left(n_{i}\right)$ :

$$
\begin{aligned}
\mathcal{L}_{\mathbf{M} 3}= & -\frac{1}{2 g_{i}^{2}} \operatorname{Tr}\left(F_{\mu \nu}^{i} F_{\mu \nu}^{i}\right)+\operatorname{Tr}\left[\left(D_{\mu} \phi_{i}\right)\left(D_{\mu} \phi_{i}\right)\right]+i \bar{\Psi} \gamma_{\mu}\left(D_{\mu} \Psi\right) \\
& -y_{i}\left[\bar{\Psi} \phi_{i} \Psi+\text { H.c. }\right]-\frac{\lambda_{i j}}{8} \operatorname{Tr}\left(\phi_{i} \phi_{i}\right) \operatorname{Tr}\left(\phi_{j} \phi_{j}\right) \\
& -\frac{\lambda_{i}}{24} \operatorname{Tr}\left(\phi_{i} \phi_{i} \phi_{i} \phi_{i}\right)
\end{aligned}
$$

where summation over $i, j=1,3$ is assumed. The gauge and Yukawa couplings are denoted by $g_{i}$ and $y_{i}$, respectively, and we have nine independent self-couplings in the model, $\lambda_{i}$ and symmetric $\lambda_{i j}$.

Finally, we consider a U(1) model with three Dirac fermions arranged as $\Psi=\left(\psi_{1}, \psi_{2}\right)$ and $\psi$. They interact with charged $(h)$ and neutral $(s)$ Higgs bosons via matrix $\left(y_{1}\right)_{i j}$, vector $\left(y_{2}\right)_{i},\left(y_{3}\right)_{i}$, and scalar $y_{4}$ Yukawa couplings:

$$
\begin{aligned}
\mathcal{L}_{\mathbf{M} 4}= & -\frac{1}{2 g^{2}}\left(F_{\mu \nu} F_{\mu \nu}\right)+\left|D_{\mu} h\right|^{2}+\frac{1}{2}\left(\partial_{\mu} s\right)^{2}+i \bar{\Psi}_{i} \gamma_{\mu}\left(D_{\mu} \Psi_{i}\right) \\
& +i \bar{\psi} \gamma_{\mu}\left(D_{\mu} \psi\right)-\left[\left(y_{1}\right)_{i j} s \bar{\Psi}_{i} P_{R} \Psi_{j}+\left(y_{2}\right)_{i} h \bar{\Psi}_{i} P_{R} \psi\right. \\
& \left.+\left(y_{3}\right)_{i} h^{*} \bar{\psi} P_{R} \Psi_{i}+y_{4} s \bar{\psi} P_{R} \psi+\text { H.c. }\right] \\
& -\frac{\lambda_{s} s^{4}}{24}-\frac{\lambda_{s h}}{2} s^{2}\left(h^{\dagger} h\right)-\frac{\lambda_{h}}{2}\left(h^{\dagger} h\right)^{2} .
\end{aligned}
$$

The U(1) charges satisfy $Q_{h}+Q_{\psi}=Q_{\Psi}$, and the sums run over $i=1,2$.
This choice of models is also motivated by the fact that we can easily implement them both in RGBeta [33] and DIANA [35]. We use the former to obtain the beta functions in terms of unknown coefficients, while the latter allows us to utilize our standard setup $[13,17]$ and compute required two- and three-point functions with FORCER [36].

To extract RG functions for gauge and Yukawa couplings, we need one-loop renormalization of the selfcouplings. We again use RGBeta to generate the necessary $Z$ factors.

Fixing coefficients.-With explicit results of calculation in models M1 (4), M2 (5), M3 (6), and M4 (7) at hand, we are in a position to apply all the collected constraints and fix all beta-function coefficients. We summarize our procedure in Table I, where we show how the number of unknowns $u_{\mathfrak{g}}$ and $u_{\mathfrak{y}}$ reduces after sequential application of available constraints. We start with WCC connecting $\mathfrak{g}^{(4)}$ and $\mathfrak{y}^{(3)}$ and interpret them as constraints on gauge beta-function coefficients. Applying further constraints, we obtain new relations (we denote the corresponding number by $\mathbf{n}$ ), and also a set of identities (the corresponding number is given by $c$ ) for cross-checking.

After matching template expressions with our toy models we are left with two unknowns in the gauge sector. In Ref. [25] the authors conjectured that $T_{I J}$ tensor entering WCC can be symmetric. This provides an additional ten constraints. We use two of them to constrain the remaining coefficients. The other eight equations become identities and, thus, verify the assumption on $T_{I J}$. It is worth noting that we use models M1-M3 to constrain all TSs but the difference $\mathfrak{y}_{172}^{(3)}-\mathfrak{y}_{173}^{(3)} \equiv 2 \delta$ in Yukawa beta function. To deal with $\delta$, we developed a special model M4, which

\begin{tabular}{|c|c|c|c|c|}
\hline \multirow{3}{*}{$\frac{\text { Type of the beta function }}{\text { Number of equations and unknowns }}$} & \multicolumn{2}{|c|}{ Gauge } & \multicolumn{2}{|c|}{ Yukawa } \\
\hline & $r=\mathbf{n}+c$ & $u_{\mathfrak{g}}$ & $r=\mathbf{n}+c$ & $u_{\mathfrak{y}}$ \\
\hline & $\ldots$ & 202 & .. & 308 \\
\hline Weyl consistency conditions & $128+0$ & 74 & $133+0$ & 175 \\
\hline Four-loop SM gauge beta functions & $63+84$ & 11 & & \\
\hline Three-loop matrix Yukawa beta functions in the SM & & & $\mathbf{1 2 8}+17$ & 47 \\
\hline Three-loop matrix Yukawa beta functions in THDM & & & $\mathbf{3 3}+213$ & 14 \\
\hline Four-loop QCD beta function for general group & $2+11$ & 9 & & \\
\hline $\mathrm{SU}\left(n_{1}\right) \times \mathrm{SU}\left(n_{2}\right)$ gauge theory (4) (M1) & $5+25$ & 4 & & \\
\hline $\mathrm{SU}(n)$ gauge theory (5) (M2) & $2+55$ & 2 & $4+76$ & 10 \\
\hline $\mathrm{SU}\left(n_{1}\right) \times \mathrm{SU}\left(n_{2}\right) \times \mathrm{SU}\left(n_{3}\right)$ gauge theory (6) (M3) & & & $\mathbf{9}+89$ & 1 \\
\hline $\mathrm{U}(1)$ gauge theory (7) (M4) & & & $\mathbf{1}+199$ & 0 \\
\hline Constraints from symmetric $T_{I J}$ & $2+8$ & 0 & & \\
\hline Final number of unknowns & & 0 & & 0 \\
\hline
\end{tabular}
explicitly confirms our initial guess that $\delta=0$.

With this procedure we fix all coefficients in gauge and Yukawa beta functions at four and three loops, respectively.

TABLE I. Reduction of the number of unknown coefficients $u_{\mathfrak{g}}$ and $u_{\mathfrak{y}}$ after sequential application of constraints. Here $r=\mathbf{n}+c$ is the rank of the system without any previous constraints included, $\mathbf{n}$ corresponds to new independent constraints, and $c$ relations are automatically satisfied due to previous steps. 
Results and discussion.-We combine the constraints from WCC [23] and our explicit computations in toy models to fix all the coefficients in the ansatz for the 4-3-2 ordering given in Ref. [25]. As an application of our general expressions, we derive all four-loop gauge beta functions in the SM extension with the arbitrary number $n_{d}$ of Higgs doublets (NHDM). We keep matrix Yukawa couplings, and by setting $n_{d}=1$ or $n_{d}=2$, we obtain the SM and THDM result. Direct computation in such a scenario would be very complicated.

Let us return to the ambiguities in theories possessing a flavor symmetry. In Refs. [25,30,32], for the coupling $y_{a i j}$ a flavor-improved, i.e., unambiguous, version $B_{a i j}$ of the Yukawa beta function $\beta_{a i j}$ is introduced

$$
B_{a i j}^{(3)}=\beta_{a i j}^{(3)}-S_{i k}^{(3)} y_{a k j}-S_{j k}^{(3)} y_{a i k}-S_{a b}^{(3)} y_{b i j} .
$$

Here $S_{i j}^{(3)}$ and $S_{a b}^{(3)}$ are three-loop quantities that can be represented as linear combinations of antisymmetric twopoint TS for fermions and scalars, respectively [25,30]. There are six coefficients $\mathfrak{f}_{1-6}^{(3)}$ entering $S_{i j}^{(3)}$ and three coefficients $\mathfrak{g}_{1-3}^{(3)}$ entering $S_{a b}^{(3)}$. All but one $\left(\mathfrak{f}_{3}^{(3)}\right)$ numerical coefficients are predicted from WCC (see Supplemental Material of Ref. [25]) in terms of $\mathfrak{g}_{i}^{(4)}$ and $\mathfrak{y}_{i}^{(3)}$ computed in this Letter. The authors of Ref. [32] calculated $\mathfrak{f}_{4}^{(3)}=-3 / 8$ and $\mathfrak{f}_{5}^{(3)}=-5 / 16$. Given our results, we provide full set of three-loop corrections to $S_{i j}$ and $S_{a b}$ :

$$
\begin{gathered}
\mathfrak{f}_{1}^{(3)}=0, \quad \mathfrak{f}_{2}^{(3)}=\frac{29}{8}-3 \zeta_{3}, \quad \mathfrak{f}_{3}^{(3)}=\frac{21}{8}-3 \zeta_{3}, \\
\mathfrak{f}_{4}^{(3)}=-\frac{3}{8}, \quad \mathfrak{f}_{5}^{(3)}=-\frac{5}{16}, \quad \mathfrak{f}_{6}^{(3)}=-\frac{7}{16} . \\
\mathfrak{g}_{1}^{(3)}=\frac{7}{2}-6 \zeta_{3}, \quad \mathfrak{g}_{2}^{(3)}=\frac{5}{8}, \quad \mathfrak{g}_{3}^{(3)}=-\frac{3}{4} .
\end{gathered}
$$

These coefficients can be tested by direct calculations along the lines of Refs. [30,32].

Conclusion.-The calculation of four-loop Gauge and three-loop Yukawa beta functions performed in this Letter complements recent six-loop results in general pure scalar theory [37], and represents the most advanced achievement in this field. The obtained TS coefficients can be incorporated into modern computer codes, giving access to a new precision level for model building. The dummy-field method (see, e.g., Ref. [38]) applied to our results provides us with scale dependence of such important quantities as fermion mass matrices.

We make all our results, including the TS coefficients and four-loop gauge-coupling beta functions in the SM, THDM, and NHDM, available as Supplemental Material [39]. We also provide a modified version of the RGBeta package with all our findings included [40].
We thank A. E. Thomsen for correspondence regarding RGBeta and Ref. [25]. We are grateful to the Joint Institute for Nuclear Research for letting us use their supercomputer "Govorun." The work of A. P. is supported by the Foundation for the Advancement of Theoretical Physics and Mathematics "BASIS." The work of A. B. is supported by the Grant of the Russian Federation Government, Agreement No. 14.W03.31.0026.

*bednya@theor.jinr.ru pikelner@theor.jinr.ru

[1] M. E. Machacek and M. T. Vaughn, Two loop renormalization group equations in a general quantum field theory. 2. Yukawa couplings, Nucl. Phys. B236, 221 (1984).

[2] M. E. Machacek and M. T. Vaughn, Two loop renormalization group equations in a general quantum field theory. 1 . Wave function renormalization, Nucl. Phys. B222, 83 (1983).

[3] M. E. Machacek and M. T. Vaughn, Two loop renormalization group equations in a general quantum field theory. 3. Scalar quartic couplings, Nucl. Phys. B249, 70 (1985).

[4] M.-X. Luo, H.-W. Wang, and Y. Xiao, Two loop renormalization group equations in general gauge field theories, Phys. Rev. D 67, 065019 (2003).

[5] A. V. Bednyakov, On three-loop RGE for the Higgs sector of 2HDM, J. High Energy Phys. 11 (2018) 154.

[6] I. Schienbein, F. Staub, T. Steudtner, and K. Svirina, Revisiting RGEs for general gauge theories, Nucl. Phys. B939, 1 (2019).

[7] L. Sartore, General RGEs for dimensionful couplings in the $\overline{\mathrm{MS}}$ scheme, Phys. Rev. D 102, 076002 (2020).

[8] G. Aad et al. (ATLAS Collaboration), Observation of a new particle in the search for the Standard Model Higgs boson with the ATLAS detector at the LHC, Phys. Lett. B 716, 1 (2012).

[9] S. Chatrchyan et al. (CMS Collaboration), Observation of a new boson at a mass of $125 \mathrm{GeV}$ with the CMS experiment at the LHC, Phys. Lett. B 716, 30 (2012).

[10] A. V. Bednyakov, B. A. Kniehl, A. F. Pikelner, and O. L. Veretin, Stability of the Electroweak Vacuum: Gauge Independence and Advanced Precision, Phys. Rev. Lett. 115, 201802 (2015).

[11] A. G. M. Pickering, J. A. Gracey, and D. R. T. Jones, Three loop gauge beta function for the most general single gauge coupling theory, Phys. Lett. B 510, 347 (2001); Erratum, Phys. Lett. B 535, 377 (2002).

[12] L. N. Mihaila, J. Salomon, and M. Steinhauser, Gauge Coupling Beta Functions in the Standard Model to Three Loops, Phys. Rev. Lett. 108, 151602 (2012).

[13] A. V. Bednyakov, A. F. Pikelner, and V. N. Velizhanin, Anomalous dimensions of gauge fields and gauge coupling beta-functions in the Standard Model at three loops, J. High Energy Phys. 01 (2013) 017.

[14] A. V. Bednyakov, A. F. Pikelner, and V. N. Velizhanin, Yukawa coupling beta-functions in the Standard Model at three loops, Phys. Lett. B 722, 336 (2013). 
[15] K. G. Chetyrkin and M. F. Zoller, $\beta$-function for the Higgs self-interaction in the Standard Model at three-loop level, J. High Energy Phys. 04 (2013) 091.

[16] A. V. Bednyakov, A.F. Pikelner, and V. N. Velizhanin, Higgs self-coupling beta-function in the Standard Model at three loops, Nucl. Phys. B875, 552 (2013).

[17] A. V. Bednyakov and A. F. Pikelner, Four-loop strong coupling beta-function in the Standard Model, Phys. Lett. B 762, 151 (2016).

[18] M. F. Zoller, Top-Yukawa effects on the $\beta$-function of the strong coupling in the SM at four-loop level, J. High Energy Phys. 02 (2016) 095.

[19] K. G. Chetyrkin and M. F. Zoller, Leading QCD-induced four-loop contributions to the $\beta$-function of the Higgs selfcoupling in the SM and vacuum stability, J. High Energy Phys. 06 (2016) 175.

[20] F. Herren, L. Mihaila, and M. Steinhauser, Gauge and Yukawa coupling beta functions of two-Higgs-doublet models to three-loop order, Phys. Rev. D 97, 015016 (2018).

[21] T. Steudtner, General scalar renormalisation group equations at three-loop order, J. High Energy Phys. 12 (2020) 012 .

[22] T. Steudtner, Towards general scalar-Yukawa renormalisation group equations at three-loop order, J. High Energy Phys. 05 (2021) 060.

[23] I. Jack and H. Osborn, Constraints on RG flow for four dimensional quantum field theories, Nucl. Phys. B883, 425 (2014).

[24] O. Antipin, M. Gillioz, J. Krog, E. Mølgaard, and F. Sannino, Standard model vacuum stability and weyl consistency conditions, J. High Energy Phys. 08 (2013) 034.

[25] C. Poole and A. E. Thomsen, Constraints on 3- and 4-loop $\beta$-functions in a general four-dimensional Quantum Field Theory, J. High Energy Phys. 09 (2019) 055.

[26] C. Poole and A. E. Thomsen, Weyl Consistency Conditions and $\gamma_{5}$, Phys. Rev. Lett. 123, 041602 (2019).

[27] F. Jegerlehner, Facts of life with gamma(5), Eur. Phys. J. C 18, 673 (2001).
[28] K. G. Chetyrkin and M. F. Zoller, Three-loop $\beta$-functions for top-Yukawa and the Higgs self-interaction in the Standard Model, J. High Energy Phys. 06 (2012) 033.

[29] J. Davies, F. Herren, C. Poole, M. Steinhauser, and A. E. Thomsen, Gauge Coupling $\beta$ Functions to Four-Loop Order in the Standard Model, Phys. Rev. Lett. 124, 071803 (2020).

[30] J.-F. Fortin, B. Grinstein, and A. Stergiou, Limit cycles and conformal invariance, J. High Energy Phys. 01 (2013) 184.

[31] A. V. Bednyakov, A.F. Pikelner, and V. N. Velizhanin, Three-loop SM beta-functions for matrix Yukawa couplings, Phys. Lett. B 737, 129 (2014).

[32] F. Herren and A. E. Thomsen, On ambiguities and divergences in perturbative renormalization group functions, J. High Energy Phys. 06 (2021) 116.

[33] A.E. Thomsen, RGBeta: A mathematica package for the evaluation of renormalization group $\beta$-functions, Eur. Phys. J. C 81, 408 (2021).

[34] P. Cvitanovic, Group theory for Feynman diagrams in nonAbelian gauge theories, Phys. Rev. D 14, 1536 (1976).

[35] M. Tentyukov and J. Fleischer, A Feynman diagram analyzer DIANA, Comput. Phys. Commun. 132, 124 (2000).

[36] B. Ruijl, T. Ueda, and J. A. M. Vermaseren, Forcer, a FORM program for the parametric reduction of four-loop massless propagator diagrams, Comput. Phys. Commun. 253, 107198 (2020).

[37] A. Bednyakov and A. Pikelner, Six-loop beta functions in general scalar theory, J. High Energy Phys. 04 (2021) 233.

[38] F. Staub, SARAH4: A tool for (not only SUSY) model builders, Comput. Phys. Commun. 185, 1773 (2014).

[39] See Supplemental Material at http://link.aps.org/ supplemental/10.1103/PhysRevLett.127.041801 for computer-readable results for TS coefficients and four-loop gauge beta functions in SM, THDM, and NHDM models.

[40] One can obtain a modified version of the RGBeta code with all the results included from, https://github.com/apik/ RGBeta. 